\title{
APORTACIÓN DE LA ANTROPOLOGÍA AL DISEÑO DE PROGRAMAS DE DESARROLLO PARA COMUNIDADES REFUGIADAS. ATENCIÓN A LAS MUJERES EN MATERIA DE SALUD Y EDUCACIÓN
}

\author{
Por \\ ISABEL Ma MARTÍNEZ PORTILLA
}

\begin{abstract}
Durante décadas se ha discutido de forma recurrente sobre lo oportuno de incluir a científicos sociales, y más concretamente a antropólogos, en las labores de diseño y puesta en marcha de programas de desarrollo comunitario. No obstante estas reticencias «teóricas», y de forma paralela a las mismas, numerosos organismos -gubernamentales y no-gubernamentales- en todo el mundo han continuado con la financiación y puesta en marcha de ambiciosos proyectos en los que participaban expertos en esas disciplinas. Ahora bien, si hacemos balance de los resultados obtenidos por algunas de esas experiencias, debemos reconocer que éstas no siempre han reportado a la comunidad sobre la que se trabajaba los beneficios que en principio se preveían; más aún, en algunos casos incluso podríamos afirmar que han provocado situaciones bastante negativas entre los pobladores de las mismas. Es necesario señalar también como en la mayoría de los casos, esos estrepitosos fracasos han sido consecuencia directa, bien de la premura y escasa planificación con que se iniciaban, bien del estrecho margen de acción que se le otorgaba al científico social que era -claro está- un simple «contratado», con unas directrices rígidas y preestablecidas desde la dirección de cada proyecto en cuestión.
\end{abstract}

"CUADERNOS DE ESTUDIOS GALLEGOS", Tomo XLVI, Fascículo 111, Santiago 1999. 
Mucho se podría añadir sobre este tema, pero no seguiré en esa línea ya que, en este breve espacio, mi intención es aportar una visión bastante más positiva de las posibilidades y ventajas que presenta la colaboración interdisciplinar en materia de desarrollo comunitario. Y de forma más concreta, de aquellas que reporta la aplicación de la Antropología -bien con la intervención directa de antropólogos, bien haciendo uso de los resultados de investigaciones previas de los mismos- en el diseño y puesta en marcha de programas en comunidades refugiadas. Este es un trabajo basado fundamentalmente en mis reflexiones y experiencias, tras varios años de trabajo de campo con refugiados y estrecho contacto y colaboración con algunas de las organizaciones que los asistían ${ }^{1}$.

En primer lugar, creo que es necesario señalar algunas de las características que a priori caracterizan a estas comunidades. Unas circunstancias que, sin duda alguna, las hace diferentes y merecedoras de una especial atención. Se trata de grupos humanos que por distintas y variadas razones se han visto obligados a abandonar sus lugares de origen, sus hogares, y buscar un nuevo espacio donde continuar sus vidas. En la mayoría de los casos son personas con escasos recursos económicos, que realizan su huida hacia el exilio llevando con ellos a sus familias y sólo algunas pertenencias. A su llegada no tienen posibilidad de trabajar, ni de acceder a la tierra, ni de obtener alimentos, ni de conseguir una vivienda digna,... no tienen ninguna posibilidad. Por ello se hace necesaria la diligente intervención desde el exterior, bien por parte del gobierno que los acoge, bien desde instituciones humanitarias internacionales. A partir de ese momento, y mientras dure su estancia en el refugio, estos extraños en tierra ajena, deberán ser asistidos de forma permanente, y en todas las áreas ${ }^{2}$. Todas estas son circunstancias muy específicas, que deben ser tenidas en cuenta a la hora de planificar programas en el interior de estas

\footnotetext{
${ }^{1}$ Durante varios años (1989-1993) he realizado campañas de trabajo de campo en el interior de comunidades guatemaltecas refugiadas en Chiapas, al sur de México. Además de este contacto directo con los refugiados, he colaborado en programas del ACNUR, la Iglesia católica y otras ONG's que desarrollan su labor en esas comunidades. Los resultados de esta investigación aparecen recogidos en mi Tesis Doctoral y en algunas publicaciones.

${ }^{2}$ Actualmente, el número de refugiados registrados por el ACNUR en todo el mundo supera los 20 millones.
}

"CUADERNOS DE ESTUDIOS GALLEGOS", Tomo XLVI, Fascículo 111, Santiago 1999. 
comunidades. Es necesario conocer la cultura de la que proceden, sus costumbres, respetar su religión, sus prácticas sociales,... Demasiados aspectos y condicionantes que no siempre son tenidos en cuenta por las organizaciones que -sin duda con el mayor interés y respeto, pero en ocasiones $\sin$ los medios y el personal adecuados- trabajan con estos grupos.

Además de todas estas consideraciones generales, y entrando ya de forma más concreta en el tema que nos interesa, es necesario señalar como en el momento de planificar y poner en marcha proyectos, bien de salud, educación, o de cualquier otra área, es necesario no olvidar un factor, sin duda alguna, de gran importancia: la existencia de una serie de rasgos diferenciadores que afectan de modo esencial la vida de hombres y mujeres en el exilio. Las mujeres, además de todas las duras circunstancias propias del refugio, sufren problemas muy concretos que las hace diferentes a sus compañeros varones y por tanto, merecedoras de unas normas de atención y protección especiales. Existen unas necesidades específicas que, aunque reconocidas desde hace tiempo por algunas Convenciones internacionales, no siempre son tenidas en cuenta ${ }^{3}$. Desde que inician la huida que las aleja de sus países, las mujeres desplazadas viven constantemente con el miedo a la violación física y de sus derechos. A su llegada a los campamentos y centros de acogida, además de las inevitables dificultades iniciales que afectan por igual a todos los refugiados, ellas deben hacer frente a problemas como la discriminación y la inseguridad sexual. Todo esto hace a las mujeres refugiadas merecedoras de unas normas de atención y protección especiales.

En la búsqueda de esa normativa específica, en 1985 , la $36^{\circ}$ sesión del Comité Ejecutivo del Alto Comisionado de Naciones Unidas para los Refugiados (ACNUR) presentó una resolución en la que se exponían una serie de recomendaciones para sus propios funcionarios y todos los Estados signatarios, con respecto a las mujeres refugiadas. Durante los debates mantenidos por el Subcomité de Protección Internacional se reconoció que las normas definidas en los instrumentos internacionales sobre los refugiados eran de carácter general, y aplicables igualmente a refugia-

\footnotetext{
${ }^{3}$ Me refiero fundamentalmente a la Convención de las Naciones Unidas sobre la eliminación de todas las formas de discriminación contra la mujer.
}

"CUADERNOS DE ESTUDIOS GALLEGOS", Tomo XLVI, Fascículo 111, Santiago 1999. 
dos de ambos sexos, y no tenían en cuenta los problemas específicos de protección a los que se enfrentan las mujeres refugiadas (Refugiados, 1988(44): 22). El ACNUR, máximo organismo en asistencia a refugiados, reconoce así que las circunstancias a las que se pueden ver expuestas las mujeres amplía el conjunto de los problemas relacionados con la protección de los mismos y que por tanto, es necesario redefinir esas garantías de acuerdo a las necesidades de las mismas.

Algunos años después, en 1991, y coincidiendo con la celebración de sus primeros cuarenta años de experiencia en el trabajo con los refugiados, el ACNUR presentó la «GUIA PARA LA PROTECCIÓN DE LAS MUJERES REFUGIADAS». En ella se reconocen las «diferencias» existentes entre hombres y mujeres en el exilio, y se proponen las soluciones y actuaciones apropiadas para cada caso. Esta Guía presenta como punto de partida las siguientes consideraciones:

«Las mujeres comparten los mismos problemas en materia de protección que el resto de los refugiados. Al igual que los demás refugiados, las mujeres necesitan protección contra el retorno forzoso a sus países de origen; seguridad contra los ataques armados y otras formas de violencia;(...) Además de estas necesidades básicas, que comparten con todos los refugiados, las mujeres y muchachas refugiadas tienen necesidades especiales de protección relacionadas con su sexo» (ACNUR, 1991b: 8).

Ahora bien, ¿cómo es posible conocer con certeza cuales son esas necesidades especiales a las que hace referencia esta Guía?, ¿de qué modo afecta a la vida de las mujeres refugiadas el que éstas no se tengan en cuenta?. Dos cuestiones de suma importancia, cuya respuesta suele estar en manos de aquellas personas que, de forma oficial o voluntaria, colaboran habitualmente con los refugiados. Y hago esta afirmación porque, aunque entiendo que en el trabajo con personas o grupos de desplazados es imprescindible seguir unas líneas programáticas muy claras y mantener unas normas, que en ocasiones pueden parecer excesivamente estrictas; además de eso, y sin tener que abandonar esa línea, creo que sería de

${ }^{4}$ Énfasis añadido.

"CUADERNOS DE ESTUDIOS GALLEGOS", Tomo XLVI, Fascículo 111, Santiago 1999. 
gran utilidad contar con la experiencia que día a día aportan los refugiados y, de forma concreta en este caso, las mujeres refugiadas. Ellas son las únicas que, «desde la otra orilla», van a ser capaces de advertir las auténticas deficiencias; las carencias y problemas urgentes cuyas soluciones, en muchos casos, son relegadas sine die por razones burocráticas o de Estado. Es de suma importancia obtener información directa de estas mujeres puesto que, sólo partiendo de sus propias experiencias, se podrán elaborar unos programas de trabajo adecuados. Proyectos acordes a sus necesidades, que mejoren los actuales niveles de asistencia y protección.

En este punto concreto, si lo que realmente nos interesa es conocer las auténticas necesidades de las refugiadas, es donde creo que la Antropología puede y debe jugar un papel muy importante. Con esta aseveración -y nada más lejos de absurdos intereses gremialistas-, quisiera recordar la posible utilidad de los resultados obtenidos tras una investigación antropológica. Durante el trabajo de campo, el antropólogo convive durante largos períodos con la comunidad que estudia, e intenta obtener de ésta toda la información posible. Con frecuencia descubre y analiza problemas, actitudes e iniciativas que hasta ese momento no habían sido observadas por otros que, desde otra perspectiva, trabajaban con esa población.

En algunos temas importantes -y sin duda, el de los refugiados lo eslos datos aportados por los antropólogos pueden llegar a ser, siempre que los Estados y Organizaciones implicadas quieran hacer correcto uso de los mismos, aplicables y positivos. La investigación puede convertirse en auténtica «Antropología Aplicada» y así beneficiar directamente, al menos a medio y largo plazo, a aquellas personas y comunidades que con anterioridad sirvieron al investigador como «objeto de estudio y análisis». Un paso mucho más importante sería, como ya señalaba páginas atrás, no sólo la utilización de esos datos obtenidos en investigaciones previas, sino una estrecha colaboración interdisciplinar a lo largo de todo el diseño y puesta en marcha de los programas de desarrollo para comunidades refugiadas. La participación directa de antropólogos podría, en muchos casos, facilitar la relación y asistencia a los refugiados. 


\section{LA PROBLEMÁTICA ESPECÍFICA DE LAS MUJERES REFUGIADAS}

Las organizaciones gubernamentales y no-gubernamentales hacen públicos, con demasiada frecuencia, datos dramáticos y alarmantes de los abusos sufridos por mujeres refugiadas. No obstante, la mayoría de los atropellos cometidos contra éstas no son denunciados por el temor de las mismas. Lo habitual es que sólo se lleguen a conocer aquellos incidentes que provocan graves consecuencias físicas, ya que las refugiadas nunca estarán dispuestas a identificarse como víctimas de violencia, sexual o de cualquier otro tipo. Por lo tanto, «... analizar el tema de las experiencias y oportunidades de las mujeres refugiadas sin tener en cuenta esos factores, sería ignorar una dimensión fundamental del dilema con que se enfrentan las mismas. Es necesario no olvidar el constante miedo hacia el abuso sexual, sea potencial o real, puesto que el mismo afectará inevitablemente a todas las acciones y percepciones de éstas mujeres refugiadas, durante su huida y búsqueda de asilo» (Aitchison, 1984. Cit en Bonnerjea, 1985).

Para muchas mujeres resulta muy difícil adaptarse a la vida en los países que las acogen, lejos de sus comunidades y su área cultural. Aquellas que llegan al refugio con sus esposos, con sus familias completas, siguen manteniendo en cierta medida el modelo de vida anterior y limitando su actividad al cuidado de los hijos y el hogar. Los hombres siguen ocupando sus roles habituales: representan a la familia en la comunidad y procuran los recursos económicos. Mucho más difícil resulta esa «nueva vida» para aquellas que llegan solas, sin la protección de la familia o la red social con la cual se identificaban en sus comunidades de origen. Estas mujeres serán inevitablemente más vulnerables a las distintas formas de intimidación sexual y explotación, sobre todo si dependen de una ayuda exterior y tienen que competir con otros por la misma.

En los campamentos y asentamientos de refugiados estas mujeres sin esposo sufren una discriminación que, en ocasiones, las aísla de la comunidad. Es muy común que se les impida participar en las actividades del campamento, que sus opiniones o problemas no sean tenidos en cuenta, encontrarlas al final de las colas durante el reparto de materiales y alimentos, etc... Por todo esto, y con el fin de dar un giro a esta injusta situación, creo que sería necesario tener en cuenta su problemática concreta concediéndoles ciertos privilegios, tanto en la distribución de los 
suministros que llegan a los asentamientos, como en el desarrollo de proyectos específicos, generadores de beneficios reales.

Tenemos múltiples ejemplos que nos demuestran que estas mujeres reciben ese trato preferente, al menos en cuanto a la distribución de alimentos y materiales en el interior de sus campamentos. Así en México por ejemplo, desde la llegada de los primeros desplazados guatemaltecos se hizo evidente que este colectivo necesitaba un tratamiento especial. Estas mujeres recibieron numerosas muestras de solidaridad por parte, tanto de sus compatriotas refugiados, como de la COMAR ${ }^{5}$, el ACNUR y otras ONG's. Respecto a esta cuestión, algunas refugiadas me relataban así lo ocurrido a su llegada a México:

«COMAR pasa a levantar los nombres, ¿cuantas viudas?, ¿cómo se llaman?. Ya hay muchas viudas y ya lo apuntan y de ahí mandan ropa para sus niños, mandan zapatos y ya preguntan que número van a calzar, y vestidos también les llega. Llegan otras cosas para las viudas, llegan ollas, llegan cubetas, platos, (...) Llegan chamarras, cobijas, y asi dan de todo antes para las viudas».

"Llegaban muchas viudas, y traían sus hijos y a sus mamás ancianas, también viudas. Ya al llegar aquí al campamento, toda la comunidad dieron la mano a estas mujeres y a otras también que no tenían sus esposos, para ayudar a levantar sus champitas donde van a vivir. Ahora trabajaban barriendo esta parte de aquí del patio central, y cuando vienen los médicos ellas se encargan de dar su alimentación a ellos. Todo estos trabajos lo hacen voluntariamente, pero siempre les llega alguna ayuda especial por parte de COMAR o del Comité de Solidaridad $»^{6}$.

Menos frecuentes son los casos donde se reconocen plenamente los derechos de estas mujeres, viudas y solas. Una de las iniciativas más excepcionales fue la iniciada hace ya algunos años por el gobierno pakistaní.

\footnotetext{
${ }^{5}$ Comisión Mexicana de Ayuda a los Refugiados. Creada en 1980 para estudiar y asistir las necesidades de los refugiados guatemaltecos que estaban llegando a sus fronteras.

${ }^{6}$ Testimonio extraído de mis conversaciones con Magdalena Tomás, refugiada guatemalteca asentada en el campamento «La Gloria», Chiapas (México). Agosto, 1991.
} 
Este estableció un campo de viudas en Nasir Bagh, donde alrededor de trescientas mujeres vivían con sus hijos. Dentro de este asentamiento, las mujeres eran libres para moverse, para ir al dispensario especial y al colegio. Allí las viudas y mujeres sin esposos esperan a que sus hijos varones crezcan y se hagan cargo del rol de cabeza de familia. Solamente entonces ellos serán una familia real ante los ojos de su comunidad (Bonnerjea, 1985:8).

Pero además de estos casos puntuales, todas las mujeres refugiadas, independientemente de su situación familiar, precisan medidas fundamentalmente prácticas. Estas son, entre otras: molinos, mejoramiento del suministro del agua, educación especial, cursos de aprendizaje,... Igualmente importante es la necesidad de incluirlas en la toma de decisiones, y en la planificación y administración de sus campamentos. Medidas estas que permitirían: la participación de las mujeres en las actividades económicas de su comunidad; mejorarían su nivel sanitario y educativo; facilitarían el acceso de las mismas a los cargos públicos; y, en definitiva, conseguirían equiparar su situación a la de sus compañeros varones. En este punto, y para conocer detenidamente algunos de los problemas más graves con los que se enfrentan cotidianamente estas mujeres y valorar las posibles soluciones, pasaré a examinar brevemente la situación de las mismas en las áreas de salud y educación.

\section{MUJERES REFUGIADAS Y SALUD}

$\mathrm{Al}$ analizar el tema de la salud de las refugiadas es necesario no olvidar que cada mujer proviene de una cultura con su propio sistema de creencias sobre la salud y la enfermedad. Sistemas en ocasiones distintos, o incluso radicalmente opuestos, a los conceptos occidentales aplicados por el personal sanitario que suele asistir los campamentos. El parto, por ej., en muchas culturas no es asunto para una relación doctor-paciente. En esas circunstancias, las mujeres confiarán en el conocimiento y las habilidades de parientes expertas o comadronas tradicionales, antes de acudir al personal médico de sus campamentos.

Asimismo, es preciso tener en cuenta que los problemas médicos planteados por las mujeres refugiadas son substancialmente diferentes a los de los hombres. Y no sólo hago referencia a los problemas ginecológicos 
y los frecuentes embarazos y lactancias, exclusivos de las mismas, sino también al estado general de nutrición, que normalmente es peor que el de los hombres. En situaciones de escasez, las mujeres suelen sufrir las mayores carencias, incluso cuando están amamantando a sus hijos recién nacidos. A esa malnutrición crónica aparecen ligadas otras enfermedades y complicaciones como tuberculosis, malaria, sarampión y parásitos, desgraciadamente muy frecuentes entre las refugiadas. Todas estas son circunstancias que nos demuestran como aún hoy, en muchas sociedades y situaciones, el valor asignado a la vida y salud de las mujeres es mucho menor que el fijado para sus compañeros varones.

La planificación familiar es otro área que plantea serias e importantes dificultades. ¿Se debe promocionar la planificación familiar entre mujeres cuya experiencia está determinada por la pérdida?. Sin duda no hay una respuesta satisfactoria y universal a esta cuestión. En muchos casos, las refugiadas deciden que no quieren colaborar en estas campañas y expresan abiertamente el deseo de tener más hijos. Esos hijos del exilio serán la única esperanza de sus familias y el futuro de sus comunidades. Por el contrario en otros casos, sobre todo en situaciones de refugiados con cierta estabilidad donde ya existen actividades generadoras de beneficios, la mayoría de las mujeres deciden que la planificación familiar es importante y necesaria. Así, y siguiendo la tendencia general de todas las sociedades, «...cuantas mas oportunidades tienen las mujeres para planificar su futuro en un adecuado contexto económico, social y político, más se interesarán por la planificación de sus familias» (Bonnerjea, 1985:12).

Además de los problemas médicos específicos de las mujeres, hay unas necesidades sanitarias generales que también se deben tener en cuenta. Los consejos en materia de higiene y cuidado de los niños, que suelen aparecer íntimamente ligados con las más profundas creencias, tradiciones culturales y pudor de las mujeres. Asimismo, la prevención y tratamiento de los problemas matrimoniales y sexuales, muy comunes en aquellas refugiadas que han sido violadas o asaltadas, serían mejor acogidos si procedieran de mujeres profesionales. Sin duda, en todas estas situaciones, la existencia de unos equipos sanitarios sensibles hacia las mujeres tendrían un importante potencial, no sólo curativo, sino también preventivo y de desarrollo. 


\section{MUJERES REFUGIADAS Y EDUCACIÓN}

El acceso a la educación representa para muchas mujeres uno de los pocos aspectos positivos de su estancia en un campamento de refugiados, pero son muchos los obstáculos. La educación de adultos es un lujo que la mayoría de los campamentos no se puede permitir y, en aquellos que existe, esta es ofrecida prioritariamente a los hombres; ya que se les supone cabeza de los núcleos familiares y por tanto, más necesitados de preparación. Además, la mayoría de las oportunidades educativas que ofrecen los campamentos son exclusivamente académicas, no técnicas o vocacionales, y esto hace difícil encontrar empleo. Las estudiantes femeninas, incluso habiendo afrontado el problema del acceso a un nivel de educación elevada, encuentran más problemas que sus compañeros varones al finalizar los estudios.

Considero necesario que los organismos gubernamentales y no-gubernamentales que colaboran con los refugiados aunen esfuerzos y reconozcan la importancia de las campañas de alfabetización de adultos. Estas beneficiarían fundamentalmente a las mujeres, puesto que ellas presentan los grados más elevados de analfabetismo. Asimismo sería muy importante capacitar a las refugiadas en determinadas áreas de trabajo -por ej. educación, sanidad, artesanías-, para qụe posteriormente desarrollaran sus conocimientos en beneficio de toda la comunidad. Como ya señalaba, en numerosas ocasiones es sumamente necesario contar con personal femenino capacitado para asistir a las mujeres refugiadas; cuánto más positivos serían los resultados de esos proyectos si el personal procede del mismo campamento, y por tanto del mismo contexto cultural.

Por último, y a modo de conclusión, sólo añadir que es necesario que tanto el ACNUR como las ONG's que colaboran con los refugiados sigan impulsando la aparición de líneas de trabajo más afines a las necesidades concretas de las mujeres refugiadas. Además de todo esto, en la práctica cotidiana, sería imprescindible contar con la experiencia aportada por las mujeres refugiadas. Sólo así se podrán elaborar y llevar a cabo futuros proyectos acordes a sus necesidades. Para conseguir esto sería importante iniciar la andadura de la interdisciplinariedad; no sólo con los antropólogos, cuyas razones ya he expuesto, sino con otros investigadores. Quizás sea hora de relegar a un segundo plano la labor de gabinete y fundamentar los programas con refugiados en el «trabajo de campo». Las 
evaluaciones que el personal de las distintas organizaciones realizan sobre las posibilidades económicas, la situación demográfica, las necesidades sanitarias, etc.... de los asentamientos de refugiados, pueden ser completadas y contrastadas con los datos obtenidos por otras vías. Datos de sociólogos, médicos, trabajadores sociales,.... y demás investigadores que han estudiado y/o convivido con estas comunidades; aunque la intención básica de sus estudios no fuera esa, ni pertenezcan de forma directa a las organizaciones «oficialmente establecidas» entre esta población. En definitiva creo que sería de suma importancia la comunicación y colaboración, para así extraer de cada disciplina todo lo que pueda tener de aplicable y positivo para los refugiados.

\section{BIBLIOGRAFÍA}

ACNUR. 1991a. ACNUR, cuarenta años al lado de los refugiados. Oficina del ACNUR para Italia. Roma (Italia).

1991b. Guía del ACNUR para la protección de las mujeres refugiadas. Ginebra.

AITCHISON, Roberta. 1984. «Reluctant Witnesses». En Cultural Survival Quarterly, (summer). Cit. en Bonnerjea, 1985.

BONNERJEA, Lucy. 1985. Shaming the world .The needs of women refugees. CHANGE (International Reports: Women and Society). Londres, Inglaterra.

KLASIANI, Shanysa Ameta. 1986. Refugee and Displaced Women in Independent African States. Economic Comission for Africa. Addis Abeba (Etiopía).

MARTÍNEZ PORTILLA, Isabel Ma . 1993. «Lucha y resistencia desde el refugio: mujeres guatemaltecas en México». En Conquista y Resistencia en la Historia de América (P. García Jordán y M. Izard, coords.). Publicaciones Universidad de Barcelona. Barcelona, pp. 375-386. 
1994 «Mujeres y hombres en el exilio: una diferencia necesaria». En Refugiados, Derecho y Solidaridad. (Pablo A. Fernández Sánchez, coord.) Publicaciones de la Universidad de Sevilla, Sevilla, pp. $113-122$.

REFUGIADOS. 1988. «Las Mujeres Refugiadas». Septiembre, nº 44. Ginebra. (Versión española a cargo del Ministerio de Asuntos Sociales). 